\title{
Cooperative Model of Bacterial Sensing
}

\author{
Yu Shi and Thomas Duke \\ TCM Group, Cavendish Laboratory, Madingley Road, Cambridge CB3 0HE, UK
}

\begin{abstract}
Bacterial chemotaxis is controlled by the signalling of a cluster of receptors. A cooperative model is presented, in which coupling between neighbouring receptor dimers enhances the sensitivity with which stimuli can be detected, without diminishing the range of chemoeffector concentration over which chemotaxis can operate. Individual receptor dimers have two stable conformational states: one active, one inactive. Noise gives rise to a distribution between these states, with the probability influenced by ligand binding, and also by the conformational states of adjacent receptor dimers. The two-state model is solved, based on an equivalence with the Ising model in a randomly distributed magnetic field. The model has only two effective parameters, and unifies a number of experimental findings. According to the value of the parameter comparing coupling and noise, the signal can be arbitrarily sensitive to changes in the fraction of receptor dimers to which ligand is bound. The counteracting effect of a change of methylation level is mapped to an induced field in the Ising model. By returning the activity to the pre-stimulus level, this adapts the receptor cluster to a new ambient concentration of chemoeffector and ensures that a sensitive response can be maintained over a wide range of concentrations.
\end{abstract}

PACS numbers: 87.10.+e, 87.22.-q, 05.20.-y

\section{INTRODUCTION}

One of the reasons why we think living things are special is that they show awareness of the environment: they respond sensitively to stimuli and can adapt to changes in the surroundings. Such biological complexity is displayed even by bacteria, which, in order to survive, have to be aware of their precarious environment where various conditions, such as nutrient and toxin levels, acidity and temperature, may change rapidly [1]. In order to discover universal principles, applicable at many levels of biological complexity, by investigating a simple system, Adler revived studies on bacterial chemotaxis which had been intensively investigated a century ago [2]. Recent genetic engineering methods have made it a paradigmatic system of cellular signaling and adaptation.

A bacterium such as Escherichia coli or Salmonella typhimurium swims smoothly by rotating a bundle of helical flagella counterclockwise, but tumbles chaotically if the flagella rotate clockwise. When it moves towards a higher concentration of attractant, such as aspartate, it tumbles less frequently. When it moves towards a higher concentration of repellent, it tumbles more frequently. So the bacterium performs a biased random walk towards an attractant and away from a repellent. This phenomenon is called chemotaxis [3]. It is mediated by receptors with extracytoplasmic sensing domains, connected by transmembrane helices to signaling domains in the cytoplasm. The receptors, which are predominantly dimeric, cluster at one pole of the cell [i which respond to different chemoeffectors but use the same signaling pathway [3,5,6,6, as shown in FIG. 1. Each receptor dimer is joined to two CheA kinase molecules, via two CheW proteins, forming a 2:2:2 complex. CheA is autophosphorylated at a rate that is greatly enhanced by the receptor. The phosphate is then passed from CheA to a regulator protein, CheY. When phosphorylated CheY diffuses to a rotatory motor, the probability of clockwise rotation of the motor, and consequently the tumbling frequency of the bacterium, increases. The binding of a chemoeffector ligand to a receptor dimer can trigger a transmembrane conformational change which regulates the autophosphorylation of CheA; attractant binding decreases the rate, while repellent binding increases it. In this way, an extra-cellular stimulus, i.e. ligand binding to a receptor, can modify the tumbling frequency. It is generally thought that there exist two stable conformational states of the receptor dimer: an "active" conformation which corresponds to a very high rate of CheA autophosphorylation; and an "inactive" conformation which corresponds to a lower rate.

An important feature of chemotaxis is that the tumbling frequency does largely depends on recent changes of the concentration of chemoeffector. This is achieved through an adaptation process, which returns the activity of the system to the pre-stimulus level after a period of time. Adaptation is assured by a feedback loop which involves another regulator protein, CheB. Like $\mathrm{CheY}$, CheB also receives a phosphate group from CheA. Phospho-CheB mediates a slow demethylation of the receptor, countering the action of CheR, which promotes methylation. Attractant binding also makes the receptor a better substrate for CheR. Since methylation enhances the autophosphorylation rate of CheA, the change in the rate of phospho-CheY production is gradually reversed by the feedback, and the the tumbling frequency ultimately returns to the pre-stimulus level. 
Each subunit of a receptor dimer consists of two helices. It has been proposed that the transmembrane signaling involves a scissor-like or pivoting motion of the pair of subunits [7, 8], or a piston-like motion involving a conformation change within just one subunit [9 11. The latter mechanism is favored by recent distance-difference analyses of the aspartate receptor, which reveal that attractant binding induces a displacement of one helix, down towards the cytoplasm, while the other three helices are not detectably perturbed [12, 13].

The chemotactic response is extraordinarily sensitive; as little as a single attractant molecule can trigger a detectable motor response [14,15,5]. Recently, Bray, Levin and Morton-Firth suggested that this sensitivity might be related to the clustering of receptors on the surface of the bacterium. Without discussing any underlying mechanism, they considered the possibility that the binding of a single molecule ligand affects the activity of a number of receptors, so that the response is augmented [16]. In this article, we present a physical model of collective signalling in a cluster of receptors. We propose that the cluster responds as an entity, as a consequence of nearest-neighbor coupling between individual receptor dimers. In our model, the influence of one dimer on another depends only on its activity, and not on whether it is liganded. Noise causes each of the receptor dimers to fluctuate between active and inactive states. When a given receptor dimer binds a molecule ligand, the probability of it being active is altered. Owing to the coupling, the probabilities of activity of adjacent receptor dimers is also modified and this effect propagates throughout the cluster. Thereby, the response to a stimulus is amplified. Moreover, ligand binding is a rapid process, while which of the receptor dimers are liganded is random, thus the above effect is averaged out, and the overall signal is a statistical average quantity as a function of the fraction of liganded receptor dimers. The model can be cast as an elementary neural network and reduced to the Ising model. Thus the paradigmatic system of cellular signaling and adaptation is related to its counterpart in statistical mechanics. The model provides a simple, unifying framework to understand a large amount of experimental data. Sensitivity to very small changes of concentration, together with the ability to respond to gradients over a broad range of concentrations, can naturally be attained. The model might be applicable to a variety of cellular signalling processes which demand a combination of low threshold of response and wide dynamic range.

The organisation of the rest of the article is as follows. In Section II, we analyse various experimental results and argue for the necessity of taking into account inter-dimer coupling, as well as noise. The neural network-like model is constructed in Section III; it is solved in Section IV, by reducing it to the Ising model with a randomly distributed magnetic field. Adaptation, and subsequent signaling of the adapted system, is specifically discussed in Section V. Section VI contains discussions and a summary.

\section{COUPLING AND NOISE}

\section{A. COUPLING}

The existence of coupling among receptor dimers is indicated by a number of experimental results. First, cooperation among receptors in signaling and adaptation is hinted at by the fact that most of the chemotactic receptors cluster into

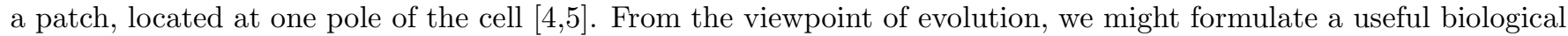
principle: An attribute that exists most probably confers advantages over possible alternatives, especially if the latter have some apparent merit. In the present case, if there were no cooperation among receptors, a uniform distribution over the surface would be optimal in efficiency for capturing molecules [17]. Since, in practice, they are found to cluster together, there is most likely an advantage due to this feature. Therefore, coupling among the receptors might well play a role in signaling and adaptation. Coupling among receptor dimers can certainly improve the sensitivity. It can amplify the signal generated by a stimulus, as has been anticipated by some authors.

Second, it has been found that signaling can occur through receptor dimers that have been genetically engineered so that one subunit lacks a signaling domain [9:18 20]. As mentioned above, a conformational change of only one subunit has been observed in the crystal structure [12,13). If the two subunits have essential differences and only one of them is involved in the transmembrane signaling, then inter-dimer coupling is inevitable to explain the experimental results on truncated subunits (with $50 \%$ probability the truncation would have been made on the signaling subunit, and no signaling would occur if the dimers act independently). However, there is also the possibility that the binding of ligand to one subunit automatically suppresses binding to the other subunit; then the transmembrane signal can always be generated with either subunit. In this case, inter-dimer coupling is not essential to explain the above experiments. Coupling is, however, necessary in order to reconcile the fact that dimers with a truncated subunit are functional with the favored mechanism of methylation, which requires interactions between two subunits of the cytoplasmic domain [5].

Third, it has been proposed, based on experiments, that at least in certain cases, receptor methylation is related to dimer-dimer interactions, i.e. methyltransferase bound to one dimer can methylate other dimers [21] [5]. Another 
support for coupling is the remarkable mobility of the P2 domain of CheA, which provides the docking site for CheY and CheB. This mobility can serve to amplify the phosphorylation signal [5]. Finally, a recent in vitro experiment by Liu et al. showed that kinase activation by a soluble signaling domain construct involves the formation of a large complex, with about 14 receptor signaling domains per CheA [23]. This appears to be a strong support for the coupling among receptor dimers in vivo.

\section{B. NOISE}

A proper consideration of noise is important for several reasons. Thermal noise is certainly a significant issue since, for biological molecules, the energy barriers between distinct conformational states are generally comparable to $k T$. Thus there is a high probability of stochastic transitions from one conformation to another. Indeed, experiments have indicated that there is considerable thermal motion in receptors. Disulphide trapping studies of the galactose/glucose binding protein have revealed spontaneous, large amplitude thermal fluctuations of the protein backbone structure $[5]$.

Moreover, noise can also provide benefits. In the absence of noise, nearest-neighbour coupling amongst receptor dimers would cause the activity to spread across the whole array and would inevitably make the response to different stimuli indistinguishable. If noise is taken into account, individual receptors flicker between active and inactive states and the overall signal is a statistical average, which naturally varies for different numbers of liganded receptor dimers.

With the consideration of noise, it is worth emphasising that the signaling process should be understood within the context of dynamic equilibrium: When the concentration of chemoeffector is stable, the conformational state and the level of methylation of each receptor dimer fluctuates microscopically, but the mean activity of the system remains unchanged. This equilibrium is shifted when the concentration of chemoeffector is changed.

\section{THE MODEL}

We study the total signal of the cluster of receptors as a quasi-equilibrium property corresponding to a certain concentration of chemoeffector and a certain level of methylation. This approach is justified by the wide separation of time scales in this system: Ligand binding and protein conformational transitions occur within milliseconds. Changes in protein phosphorylation occur on a time scale of $\sim 0.1 s$. The much slower adaptation process, associated with modulations of methylation level, is on a scale of minutes [3,22].

The quasi-equilibrium state of a dynamics is determined by the minimum of a noisy "energy function" (a Lyapunov function). This "energy" is not necessarily the actual physical energy, since it may describe an effective dynamics that "renormalizes" the underlying chain of physical processes. Similarly, the noise may not be due only to the temperature, but in the present case it mostly is. Such a description, which reduces degrees of freedom, is especially effective when the underlying physical processes are complicated, or unclear in detail. A typical example is Hopfield's neural network model [24,25]. Here we adopt this approach for chemotactic signaling, but with a different interpretation and with the emphasis placed on the determination of the equilibrium activity as a function of the external stimuli.

Consider a lattice of receptors, whose basic unit is the receptor dimer, or equivalently, the whole receptor-CheWCheA dimer complex. Initially, we consider a system that has adapted to zero concentration of the chemoeffector, and investigate the response when the concentration changes so that a fraction $c$ of receptor dimers are bound to chemoeffector molecule ligands. The state of a receptor is a function of the effect of ligand binding and the states of the neighboring receptor dimers. Characterizing the state of receptor dimer $i$ by a variable $V_{i}$ (according to recent observation, it is the vertical position of one of four helices, but we are open to possible re-interpretation according to experimental findings), and the effect of the ligand binding to receptor dimer $i$ by $H_{i}$, most generally we have

$$
V_{i}=V_{i}\left(\left\{V_{j \neq i}\right\},\left\{H_{j}\right\}\right)
$$

where $\left\{H_{j}\right\}$ denotes the set of $H_{j}$ for $j=1,2, \cdots,\left\{V_{j \neq i}\right\}$ denotes the set of all $V_{j}$ for $j \neq i$. The natural assumption is that $V_{i}$ is affected only by $H_{i}$ and the states of the nearest neighbours. Furthermore, for the two-state model, in which $V_{i}$ has only two possible values, $V^{0}$ or $V^{1}$, the McCulloch-Pitts threshold model 26, 25] is a natural assumption. Thereby

$$
V_{i}=\psi\left(\sum_{j} T_{i j} V_{j}+H_{i}-U_{i}\right), \text { with } \psi(x)= \begin{cases}V^{1} & \text { if } x>0 \\ V^{0} & \text { if } x \leq 0\end{cases}
$$


where $U_{i}$ is a threshold value, and $T_{i j}$ describes the coupling among receptor dimers, which is assumed to be nonzero only for nearest neighbours. We adopt the convention that $V^{0}$ is the active conformation and $V^{1}$ is the inactive one. Then $H_{i}>0$ for attractant binding, which tends to inactivate receptors and depress the autophosphorylation rate of CheA, thereby decreasing the frequency of tumbling. Conversely, $H_{i}<0$ for repellent binding.

It is well known that if $T_{i j}=T_{j i}$ and $T_{i i}=0$, clearly valid in the present situation, the dynamics is determined by a Lyapunov function (or Hamiltonian) [25],

$$
\mathcal{H}=-\sum_{<i j>} T_{i j} V_{i} V_{j}-\sum_{i} H_{i} V_{i}+\sum_{i} U_{i} V_{i},
$$

where $\langle i j\rangle$ represents pairs of nearest neighbours. Taking into account the noise, which induces a state distribution which is nearly a Boltzmann distribution [25], the problem reduces to the statistical mechanics of a system with the above Hamiltonian.

In the simplest interpretation, the noise is purely thermal, $\beta=1 / k T$, and Eq. (3) may be identified as the effective physical energy. According to recent observation, $V^{0}$ and $V^{1}$ are the two stable positions of one of the four helices. Therefore $H_{i}$ and $T_{i j} V_{j}$ are forces due to ligand binding and coupling, respectively. The "Zeeman energy" dependent on ligand binding is due to the free energy exchange with bound ligand. Similarly, the coupling energy is due to free energy exchange with the cytoplasm or membrane, which mediates the effective coupling.

Eq. (3) may be transformed to the "spin" representation by writing $S_{i}=2\left(V_{i}-V^{0}\right) / \Delta V-1$, where $\Delta V=$ $\left(V^{1}-V^{0}\right)$. Then

$$
\mathcal{H}=-\sum_{<i j>} J_{i j} S_{i} S_{j}-\sum_{i} B_{i} S_{i}+\mathcal{H}_{1}+E_{0},
$$

where $J_{i j}=T_{i j} \Delta V^{2} / 4, B_{i}=H_{i} \Delta V / 2$, and $E_{0}$ is a constant given a distribution of $\left\{B_{i}\right\} . \mathcal{H}_{1}$ is a "Zeeman energy" due to an effective "magnetic field" independent of $\left\{B_{i}\right\}$, which determines the equilibrium configurations in the absence of $\left\{B_{i}\right\}$, i.e. without ligand binding. Without loss of generality, we set $\mathcal{H}_{1}=0$. Thus in the absence of $\left\{B_{i}\right\}$, and if the noise is sufficiently high, $S_{i}$ is equally distributed between 1 and -1 , and the "magnetization" is zero. In other words, it is assumed that there is no energy difference between the active $\left(S_{i}=-1\right)$ and inactive $\left(S_{i}=1\right)$ conformations for an isolated, unliganded receptor dimer $i$. The physics does not change if this difference is set to be nonzero. Ligand binding shifts the energy difference to $2 B_{i}$.

We have now reduced the model to an Ising model. The activity of the array of receptors corresponds to the average magnetization of a lattice of spins, and ligand binding of a receptor dimer corresponds to a local magnetic field at a lattice site: $B_{i}=B$ if receptor dimer $i$ binds a chemoeffector ligand, otherwise $B_{i}=0$. If the fraction of liganded receptor dimers is $c$, then the value of $B_{i}$ is randomly distributed between $B$ and 0 with probability

$$
p\left(B_{i}\right)=c \delta\left(B_{i}-B\right)+(1-c) \delta\left(B_{i}\right) .
$$

This Ising model in a field bimodally distributed between 0 and $B$ is simpler than the so-called "random-field Ising model" [27 30, in which the possible values of the field are symmetric with respect to zero, and nontrivial results arise due to the fluctuation of the fields. In our case, the average of the field is nonzero, so there is a long-range order simply as the result of the explicit symmetry breaking. For Eq. (价, $\bar{B}_{i}=c B$, where the overbar denotes the average over disordered configurations. The fluctuation of the random distribution is $\Delta B_{i}=\sqrt{c(1-c)} B$. Consider the formation of a domain of size $L$ in the ferromagnetic ground state. According to the central limit theorem, the average Zeeman energy is $\sim L^{d} c B$, much larger than its fluctuation, which is $\sim L^{d / 2} \sqrt{c(1-c)} B$. Therefore the energy gain is always positive, and the fluctuation of the field cannot destroy long-range order.

\section{SOLUTIONS OF THE MODEL}

The two-state model, which has been reduced to the Ising model in a randomly distributed field, as described by Eqs. (画) and (5), can be solved by the mean-field method. One may obtain the result simply by considering that the average magnetization, $m=\overline{\left\langle s_{i}\right\rangle}$, where $\langle\cdots\rangle$ denotes the thermodynamic average, is determined by the local field $B_{i}^{\prime}=\sum_{j} J_{i j} m+B_{i}$ with the random distribution. Alternatively one may first obtain the free energy using the replica method, then calculate the average magnetization [28,29. It is found that $m$ is the root of the equation

$$
\begin{aligned}
m & =\overline{\tanh \left(\beta \nu J m+\beta B_{i}\right)} \\
& =\frac{2 c}{1+\exp [-2(\beta \nu J m+\beta B)]}+\frac{2(1-c)}{1+\exp (-2 \beta \nu J m)}-1,
\end{aligned}
$$


while the the noisy Lyapunov function is

$$
\left.F=\frac{1}{2} \nu J m^{2}-\frac{1}{\beta}\{c \ln [2 \cosh (\beta \nu J m+\beta B))]+(1-c) \ln [2 \cosh (\beta \nu J m)]\right\}+E_{0} .
$$

Here, $J_{i j}$ has been assumed to have a single value $J$ for nearest neighbors, $\nu$ is the number of nearest neighbors, and $\beta$ is a characterization of the noise.

The relation between the chemoeffector concentration and the activity of the system is now reduced to the $m$ versus $c$ relation, determined by Eq. (7), since the activity of the system, here defined as the fraction of receptor dimers in the active state is $A=(1-m) / 2$, and the pure response to the stimulus, i.e. the change of the activity, is $\Delta A=m / 2$. Although Eq. (7) may possibly have more than one solution, the one corresponding to the lowest $F$ is what we need. Approximate analytical solutions may be found in limiting cases,

$$
m \approx \begin{cases}\frac{\beta c B}{1-\beta \nu J} & \text { if } \beta \rightarrow 0 \\ 1-2(1-c) \exp (-2 \beta \nu J)-2 c \exp [-2(\beta \nu J+\beta B)] & \text { if } \beta \rightarrow \infty \text { and } B>0 \\ -1+2(1-c) \exp (-2 \beta \nu J)+2 c \exp -2[\beta \nu J+\beta|B|) & \text { if } \beta \rightarrow \infty \text { and } B<0\end{cases}
$$

In general, the solution can only be obtained numerically. It can be seen that there are actually only two effective parameters in this model; one is $\alpha=\beta \nu J$, the other is $\gamma=\beta B$. Owing to symmetry, it suffices to give results for $B>0$. Solutions for typical values of parameters are shown in FIG. 2. First we choose $\alpha=0.1,0.8,1.2$; then for each $\alpha$, the dependence of $m$ on $c$ is determined for $\gamma=0.01,0.01,0.1,1,10,100$. Note that for $c=0$, i.e., the Ising model without a magnetic field, $\alpha=1$ is the critical value dividing the "paramagnetic" phase, where $m(c=0)=0$, and the "ferromagnetic" phase, where there is a "spontaneous magnetization", $m(c=0) \neq 0$.

The quantitative measure of sensitivity, denoted by $S$, is half of the slope at $c=0$ :

$$
\begin{aligned}
S & =\left.\frac{\partial(m / 2)}{\partial c}\right|_{c=0} \\
& =\frac{\frac{1}{1+\exp \left[-2\left(\beta \nu J m_{0}+\beta B\right)\right]}-\frac{1}{1+\exp \left(-2 \beta \nu J m_{0}\right)}}{1-\frac{4 \beta \nu J \exp \left(-2 \beta \nu J m_{0}\right)}{\left[1+\exp \left(-2 \beta \nu J m_{0}\right)\right]^{2}}},
\end{aligned}
$$

where $m_{0}=m(c=0)$. It is clear that $S$ can be made arbitrarily large by choosing appropriate value of $\beta \nu J$ so that the denominator in the above expression is arbitrarily close to 0 . For $m_{0}=0$,

$$
S=\frac{\frac{1}{1+\exp (-2 \beta B)}-\frac{1}{2}}{1-\beta \nu J},
$$

which is directly tuned by the difference between $\beta \nu J$ and 1 , which is the critical value of phase transition for $c=0$. The case with $m_{0} \neq 0$ is less favored since the range of possible $m$ for different $c$ could be diminished, making it more difficult to distinguish between different stimuli. Moreover, the sign of $m_{0}$ would be determined by that of the previous $B$, conflicting the fact that the pre-stimulus level is fixed.

For a given $\alpha, S$ also increases with $\gamma=\beta B$, but with an upper bound. The fact that $\partial m / \partial \gamma \rightarrow 0$ when $\gamma \rightarrow \infty$ indicates that, if ligand binding has a strong enough effect, the response is independent of the exact value of $\gamma$. This provides a sort of stability for the effect of ligand binding.

Thus good sensitivity requires fine tuning of the coupling: the greater the sensitivity demanded by the bacterium, the more accurately $\alpha=\beta \nu J$ has to be controlled. But $\gamma=\beta B$ may vary widely without considerably affecting the response. This is reasonable, since the temperature range suitable for bacterial survival is rather restricted and, for a given bacterium, $\nu J$ is a structural property, which could be optimized during evolution. On the other hand, the effect of ligand binding, $B$, depends on the external stimulus, which may vary considerably.

As an exercise, our model may be applied to the puzzling situation in which both attractants and repellents are present [2]. In this case,

$$
p\left(B_{i}\right)=c_{r} \delta\left(B_{i}-B_{r}\right)+c_{a} \delta\left(B_{i}-B_{a}\right)+\left(1-c_{r}-c_{a}\right) \delta\left(B_{i}\right),
$$

where $c_{r}$ and $c_{a}$ are the concentrations of the repellents and attractants, respectively, and $B_{r}$ and $B_{a}$ are respectively the measures of the attractant and repellent binding. Obviously the activity is dependent on both $c_{r}$ and $c_{a}$. 


\section{ADAPTATION}

Now we incorporate into this model the delayed adaptation due to the change of methylation level. This may be achieved through an induced "field" with an opposite sign to that associated with ligand binding, so that the "magnetization" returns toward the pre-stimulus level. This assumption for the additivity of the effect of ligand binding and that of the change of methylation level is supported by the finding that in a receptor there is a region which gathers, integrates, and interprets the multiple inputs transferred by the transmembrane signaling domain and the methylated side chains, then transmits an output signal to the kinase regulation machinery [5].

Two points should be made clear. First, the time scale of the change of the level of methylation of the whole system is much longer than the microscopic time scale, so the "magnetization" can still be obtained as the equilibrium property of the noisy Lyapunov function, which quasi-statically changes with the level of methylation. Second, since the ligand binding occurs on a time scale much shorter than the time needed for adaptation to be completely achieved, we cannot simply change the value of $B$, but must introduce another "field". We denote this "induced field" by $\left\{M_{i}\right\}$, with the distribution

$$
p\left(M_{i}\right)=c_{m} \delta\left(M_{i}-M\right)+\left(1-c_{m}\right) \delta\left(M_{i}\right),
$$

where $c_{m}$ is the fraction of the receptor dimers whose original level of methylation is modified. The sign of $M$ is opposite to that of $B$. Thereby the net field is $D_{i}=M_{i}+B_{i}$ with the distribution

$$
\begin{aligned}
p\left(D_{i}\right)= & c c_{m} \delta\left(D_{i}-B-M\right)+c\left(1-c_{m}\right) \delta\left(D_{i}-B\right) \\
& +(1-c) c_{m} \delta\left(D_{i}-M\right)+(1-c)\left(1-c_{m}\right) \delta\left(D_{i}\right) .
\end{aligned}
$$

The equilibrium state can be obtained by replacing $B_{i}$ in Eq. (4) with $D_{i}$. Adaptation is taking place if $c_{m}$ and/or $M$ vary slowly with time. This gives rise to a time-dependent "magnetization", which may return to zeo. To get a quantitative impression, by adopting the high noise limit $\beta \rightarrow 0$, it may be estimated that when $c_{m} B+c M=$ 0 , adaptation is completed; the "magnetization" returns to zero. Here, we shall simply assume that a molecular mechanism exists which ensures that the state of zero "magnetization" is an attractor of the dynamics, so that adaptation is exact. A more precise study of the adaptation process will be reported in the future.

Once the system has adapted, suppose that the concentration subsequently changes from $c$ to $c+\delta c$. One can obtain the new activity by substituting in Eq. (14) $c+\delta c$ for $c$, and the values of $c_{m}$ and $M$ at which the adaptation was completed. In general, what is most important is the change of fraction of liganded receptor dimers since the last adaptation. Under high temperature approximation, the result is Eq. (9) with $c$ replaced by $\delta c$. Moreover, it can be seen that if $\delta c$ is negative, i.e if chemoeffector is removed, the effect is similar to the addition of a chemoeffector whose "field" has opposite sign. Therefore the removal of attractant is equivalent to repellent binding, and vice versa. This has indeed been observed in experiments [22].

\section{SUMMARY AND DISCUSSIONS}

In this article, we analyse relevant experimental results and draw the conclusion that both inter-dimer coupling and noise are crucial in the mechanism of chemotactic signaling and adaptation. The ratio between their measures, $\alpha=\beta \nu J$ is one of the two effective parameters in the cooperative model we construct. A second parameter is the ratio between the measure of the effect of ligand binding and that of the noise, $\gamma=\beta B$. The essential features due to the balance of coupling and noise are well captured by the paradigmatic model of statistical mechanics, the Ising model. We made an attempt to map the underlying mechanism of collective effects in chemotactic signaling to the Ising model in a randomly distributed field, with the distribution reflecting the ligand occupancy. To complete the mapping, we adopted the basis of Hopfield's neural network model. The great difference between time scales of the various chemical and mechanical processes makes it feasible to obtain the signaling level as a quasi-equilibrium property of a noisy Lyapunov function. This Lyapunov function describes the dynamics "renormalizing" underlying complexity.

Our model provides the following picture. An individual receptor dimer has two stable conformational states, an active one that corresponds to a high rate of CheA autophosphorylation, and an inactive one that corresponds to a low rate. Noise gives rise to a distribution between these states and the partition is influenced both by ligand binding and by the conformational states of the neighboring receptor dimers. In the simplest interpretation, the noise is purely thermal, $\beta=1 / k T, 2 B$ corresponds to the shift of the energy difference between active and inactive states induced by ligand binding, and $J$ measures the effective coupling energy between neighboring receptor dimers. The activity of the receptor cluster is a statistical average quantity. A change in the fraction of liganded receptor dimers causes 
the total activity to change from the pre-stimulus level. But the level of methylation also changes, on a slower time scale. This causes an effect opposite to that induced by ligand binding. Consequently, the total activity ultimately returns to the pre-stimulus level.

The coupling between receptor dimers naturally provides the sensitivity to small stimuli observed in experiments. Additionally, the noise makes the response to different values of concentration changes distinctive. Sensitivity to small changes in the environment requires fine tuning of the parameter $\alpha$, but $\gamma$ may vary without considerably affecting the response. The equivalence between the removal of attractant and the addition of repellent, or vice versa, has a natural explanation.

Among problems for further investigation are the effects of finiteness of the number of receptor dimers, potential randomness in the coupling, and features that might be lost in the mean field solution. Correlation between $B_{i}$, or $M_{i}$, at different sites $i$ is also a possibility and might have useful consequences. The finite-size effect and the "random field" due to the change of methylation level may destroy the "spontaneous magnetisation" that exists for $\alpha>1$, thus relaxing the constraint on the precision to which $\alpha$ must be specified to give high sensitivity. The mean-field solution is least accurate when $c \rightarrow \frac{1}{2}$, since the fluctuation of the field is $\sqrt{c(1-c) B}$, which increases to the greatest as $c \rightarrow \frac{1}{2}$. Thus maybe the sensitivity is lower at moderate values of the occupancy $c$, than at the extremes $c \rightarrow 0$ and $c \rightarrow 1$. However, this is not necessarily a limitation. The fractional occupancy $c$ is related to the ambient concentration of ligand $[L]$ by

$$
c=\frac{[L]}{[L]+K_{d}}
$$

where $K_{d}$ is the dissociation constant. Thus

$$
\delta c=\frac{[L] K_{d}}{\left([L]+K_{d}\right)^{2}} \frac{\delta[L]}{[L]}=c(1-c) \frac{\delta[L]}{[L]} .
$$

Given that the bacterium probably needs to detect a relative change in concentration, $\frac{\delta[L]}{[L]}$, we see that the greatest sensitivity to a change in occupancy is demanded when $c \rightarrow 0$ or $c \rightarrow 1$, and the least when $c \rightarrow \frac{1}{2}$.

It is well known that the the two-state threshold neural network model is equivalent to a model with continuous variables in the high gain limit [24], with the Lyapunov function

$$
\mathcal{H}=-\sum_{<i j>} T_{i j} V_{i} V_{j}+\sum_{i} \frac{1}{R_{i}} \int_{0}^{V_{i}} g_{i}^{-1}(V) d V-\sum_{i} H_{i} V_{i}
$$

with $u_{i}=g_{i}^{-1}\left(V_{i}\right)$ determined by

$$
C \frac{d u_{i}}{d t}=\sum_{<i j>} T_{i j} V_{j}-\frac{u_{i}}{R_{i}}+H_{i},
$$

where $u_{i}$ is interpreted as the soma potential, while $C$ is the input capacitance of the cell membrane. When Eqs. (17) and (18) are adopted for the network of chemoreceptor dimers, $V_{i}$ is a variable characterizing the stable conformation, i.e. the (vertical) position of the mobile helix of the receptor dimer, and $u_{i}$ is the instantaneous position. Thus Eq. (18) could be the equation of motion describing the transient process of the movement of the mobile helix, in response to a force $H_{i}$ generated by ligand binding, as well as forces due to couplings with the neighboring receptor dimers. Of course, whether modification of (18) is necessary depends on future experimental results.

According to this interpretation, by measuring the force generated by ligand binding, $H$, and the displacement of the mobile (signalling) helix $\Delta V=\left(V^{1}-V^{0}\right)$, one may obtain the parameter $B=H \cdot \Delta V / 2$. Note that $H \cdot \Delta V$ is the work done by the force $H$, consistent with the identification of $2 B$ with the shift in energy difference caused by ligand binding. Similarly, $4 J / \Delta V=T_{i j} \Delta V$ is the force generated by the conformational change of one of the nearest neighbours. To make a rough estimation, we take typical values $\alpha=0.5, \gamma=5$ and $1 / \beta \approx 4 p N \cdot n m$ (assuming that the noise is purely thermal). Then $B \approx 20 p N \cdot n m, \nu J \approx 2 p N \cdot n m$. The measured displacement is $0.16 n m$ [12. It is found that the force resulting from ligand binding is about $250 \mathrm{pN}$ and the force due to coupling between a pair of nearest neighbours is about $10 p N$. These orders of magnitude are quite reasonable.

Since the continuum model can be realized in electric circuits, more insights might be provided from the viewpoint of system control, where negative feedback has been well studied. On the other hand, the analogy with the neural network model is possibly more than a mathematical one. From the viewpoint of evolution, there are common features between bacterial sensing and sensing of higher animals. Perhaps a primitive or ancestral neural network works in 
chemotaxis. Adler writes: "The basic elements that make behaviour possible in higher organisms are also present in a single bacterial cell; they are sensory receptors, a system that transmits and processes sensory information and effectors to produce movement. Whether the mechanisms of any of these elements in bacterium are similar to those in more complex organisms remains to be established" [2]. Margulis thinks: "Thought and behaviour in people are rendered far less mysterious when we realize that choice and sensitivity are already exquisitely developed in the microbial cells that became our ancestors" [31. We hope our approach is a small step in addressing these issues.

\section{ACKNOWLEDGMENTS}

We are very grateful to D. Bray for valuable discussions and comments, and for critically reading the manuscript. Y.S. also thanks G. Fath and P. Littlewood for discussions.

FIG. 1. A schematic illustration of the chemotactic signaling pathway.

FIG. 2. The solution of the two-state model: "magnetization" $m$ as a function of the fraction $c$ of liganded receptor dimers. Here we assume there was no ligand bound previously. The three figures are for three typical values of the parameter $\alpha=\beta \nu J$ : (a) $\alpha=0.1$; (b) $\alpha=0.8$; (c) $\alpha=1.2$. In each figure, different plots are for different values of the parameter $\gamma=\beta B$ : $\diamond: \gamma=0.01 ;+: \gamma=0.1 ; \square: \gamma=1 ; \times: \gamma=10 ; \triangle: \gamma=100$. Note that the critical pont, which separates "ferromagnetic" and "paramagnetic" phases, is $\alpha=1$.

[1] J.S. Parkinson and E.C. Kofoid, Annu. Rev. Genet. 26, 71 (1992).

[2] J. Adler, Sci. American, 234(4), 40 (1976).

[3] J. Stock and M. Surette, in Escherichia coli and Salmonella typhimurium: Cellular and Molecular Biology, ed. F.C. Neidhardt, (ASM, Washington, 1996).

[4] J.S. Parkinson, J. S. and D.F. Blair, Science 259, 1701 (1993); M.R.K. Alley, J.R. Maddock, and L. Shapiro, ibid. 1754 (1993); J.R. Maddock and L. Shapiro, ibid. 1717 (1993).

[5] J.J. Falke et al., Annu. Rev. Cell Dev. Biol. 13, 457 (1997).

[6] D.F. Blair, Annu. Rev. Microbiol. 49489 (1995).

[7] M.V. Milbourn et al., Science 254, 1342 (1991).

[8] A.G. Cochran and P.S. Kim, Science 271, 1113 (1996).

[9] D.L. Milligan and D.E. Koshland Jr., Science 254, 1651 (1991).

[10] A. Pakula and M. Simon, Nature, 355496 (1992).

[11] B.L. Stoddard, J.D. Bui, and D.E. Koshland Jr., Biochemistry 31, 11978 (1992).

[12] S. Chervitz and J.J. Falke, Proc. Natl. Acad. Sci. USA 93, 2545 (1996).

[13] A.G. Hughson and G.L. Hazelbauer, Proc. Natl. Acad. Sci. USA 93, 11546 (1996).

[14] S.M. Block, J.E. Segall, and H.C. Berg, J. Bacterial 154, 312 (1983).

[15] J.E. Segall, S.M. Block, and H.C. Berg, Proc. Natl. Acad. Sci. USA 83, 8987 (1986).

[16] D. Bray, M.D. Levin, and C.J. Morton-Firth, Nature, 393, 85 (1998).

[17] H.C. Berg and E.M. Purcell, Biophys. J. 20, 193 (1977).

[18] P.J. Gardina and M.D. Manson, Science 274, 425 (1996).

[19] I. Tatsuno, M. Homma, K. Oosawa, and I. Kawagishi, Science 274, 423 (1996).

[20] J. Stock, Science 274, 370 (1996).

[21] J.R. Wu, J.Y. Li, G.Y. Li, D.G. Long, and R.M. Weis, Biochemistry 35, 4984 (1996).

[22] M.S. Springer, M.F. Goy, and J. Adler, Nature 280, 279 (1979).

[23] Y. Liu, M. Levit, R. Lurz, M.G. Surrette, and J. Stock, The EMBO Journal 16, 7231 (1997).

[24] J.J. Hopfield, Proc. Natl. Acad. Sci. USA 81, 3088 (1984); Phys. Today, 47(2), 40(1994).

[25] D.J. Amit, Modeling Brain Function (Cambridge University Press, Cambridge, 1989).

[26] W.S. McCulloch and W.A. Pitts, Bull. Math. Biophys. 5, 115 (1943).

[27] Y. Imry and S.-K. Ma, Phys. Rev. Lett. 35, 1399 (1975).

[28] T. Schneider and E. Pytte, Phys. Rev. B, 15, 1519 (1977).

[29] A. Aharony, Phys. Rev. B 18, 3318 (1978). 
[30] D.P. Belanger and A.P. Young, J. Mag. Mag. Materials 100, 272 (1991).

[31] L. Margulis, in The Third Culture, ed. J. Brockman, (Simon \& Schuster, NewYork, 1995). 
Receptor dimer

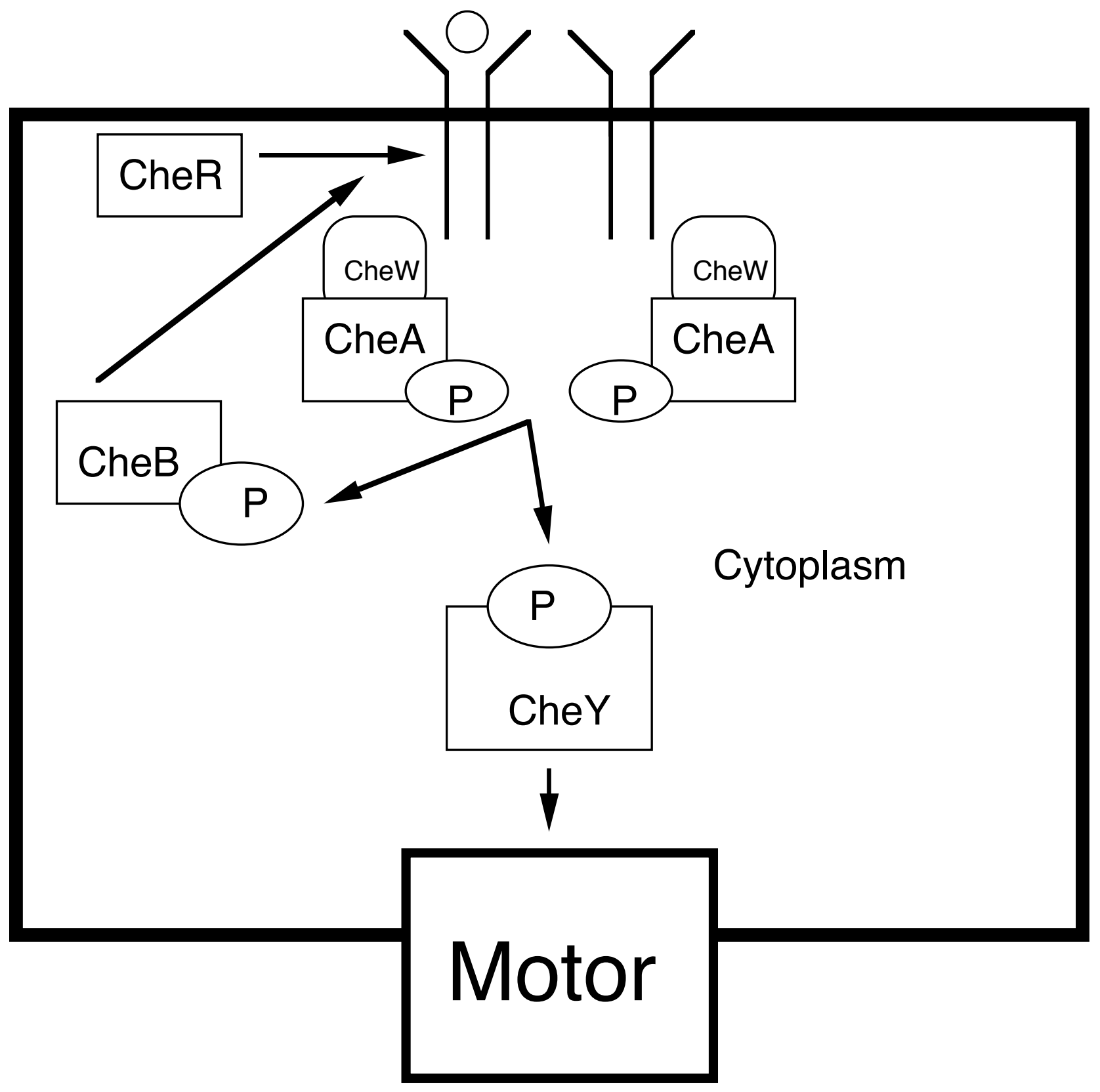




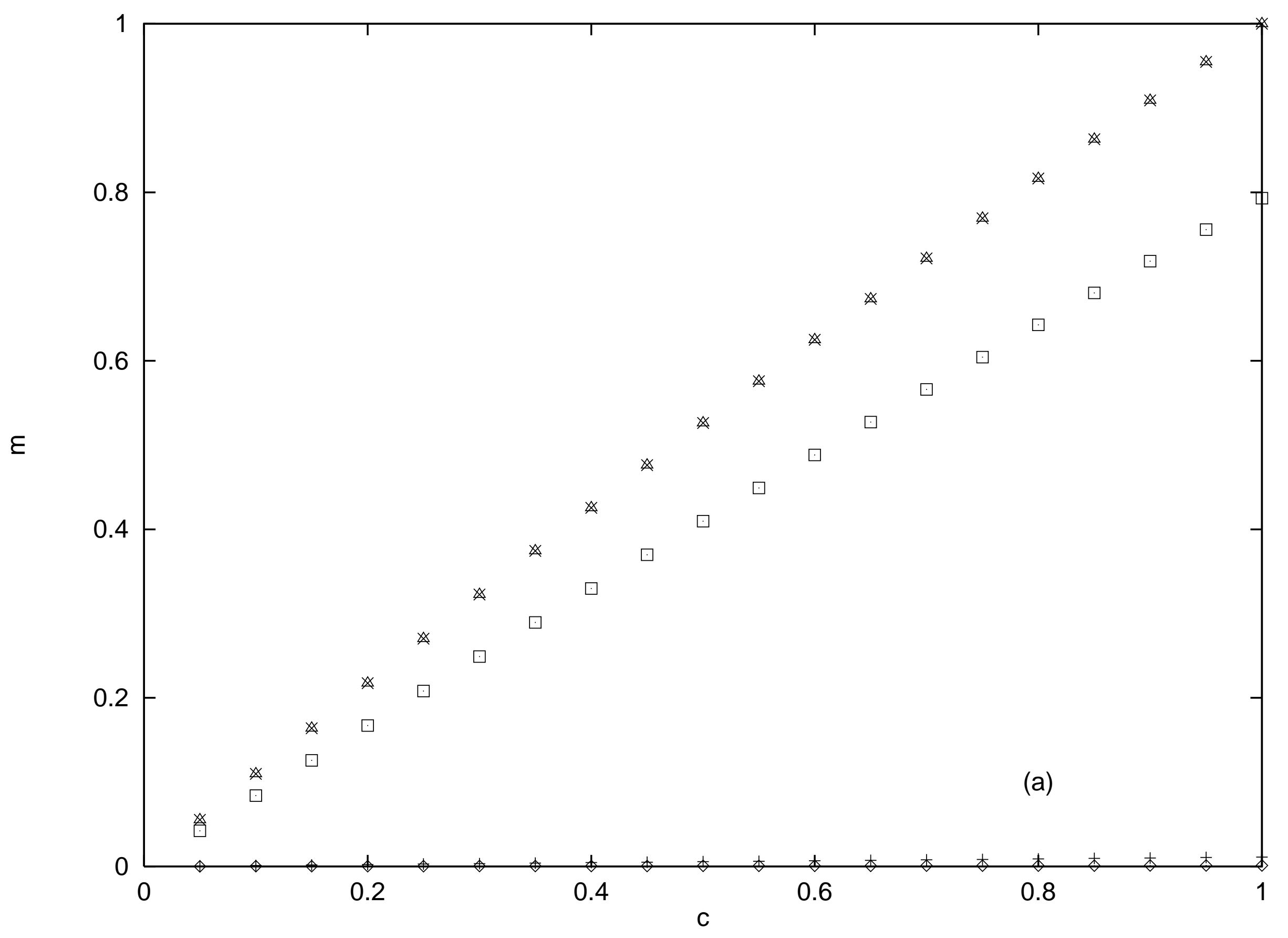




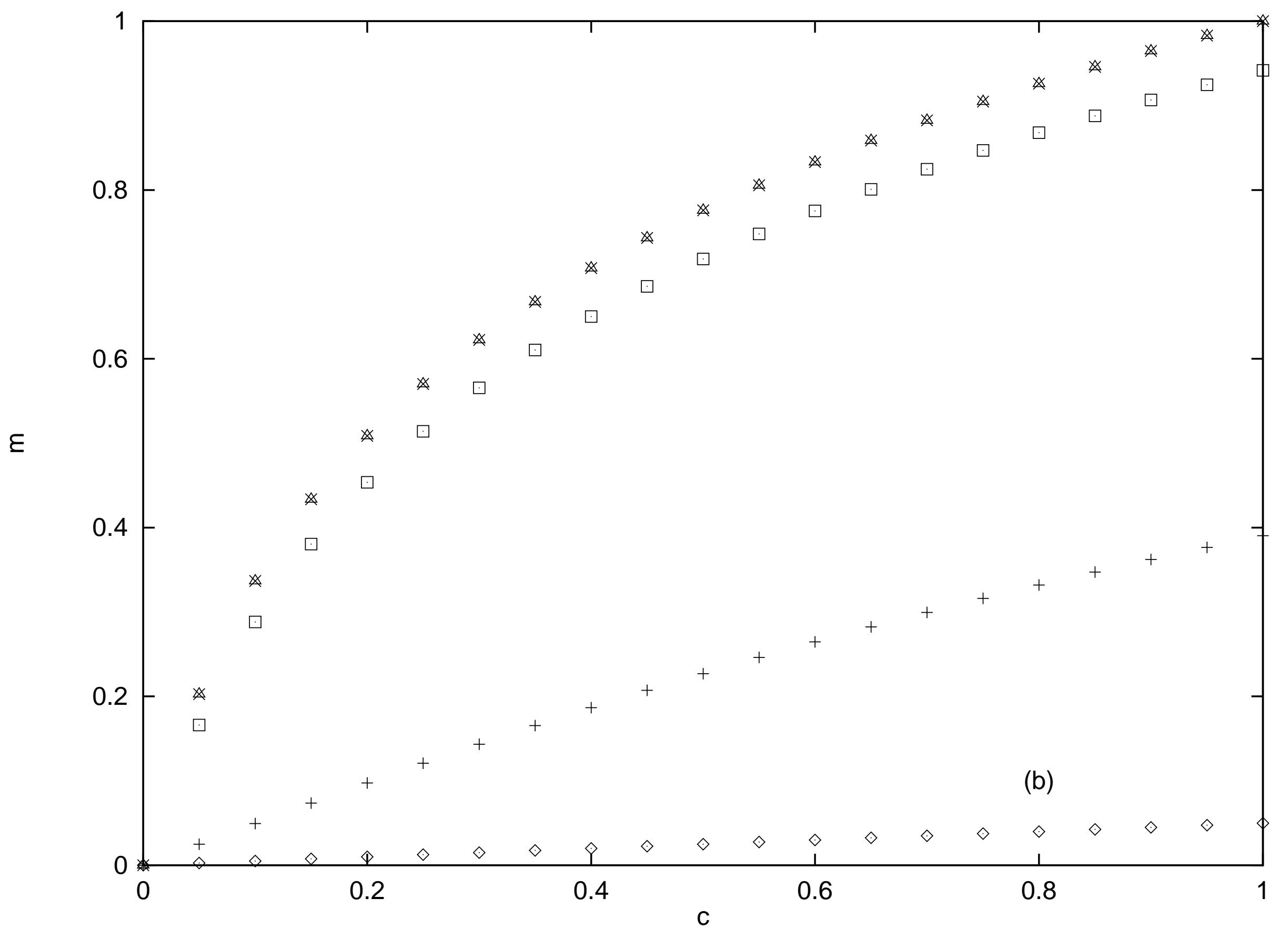




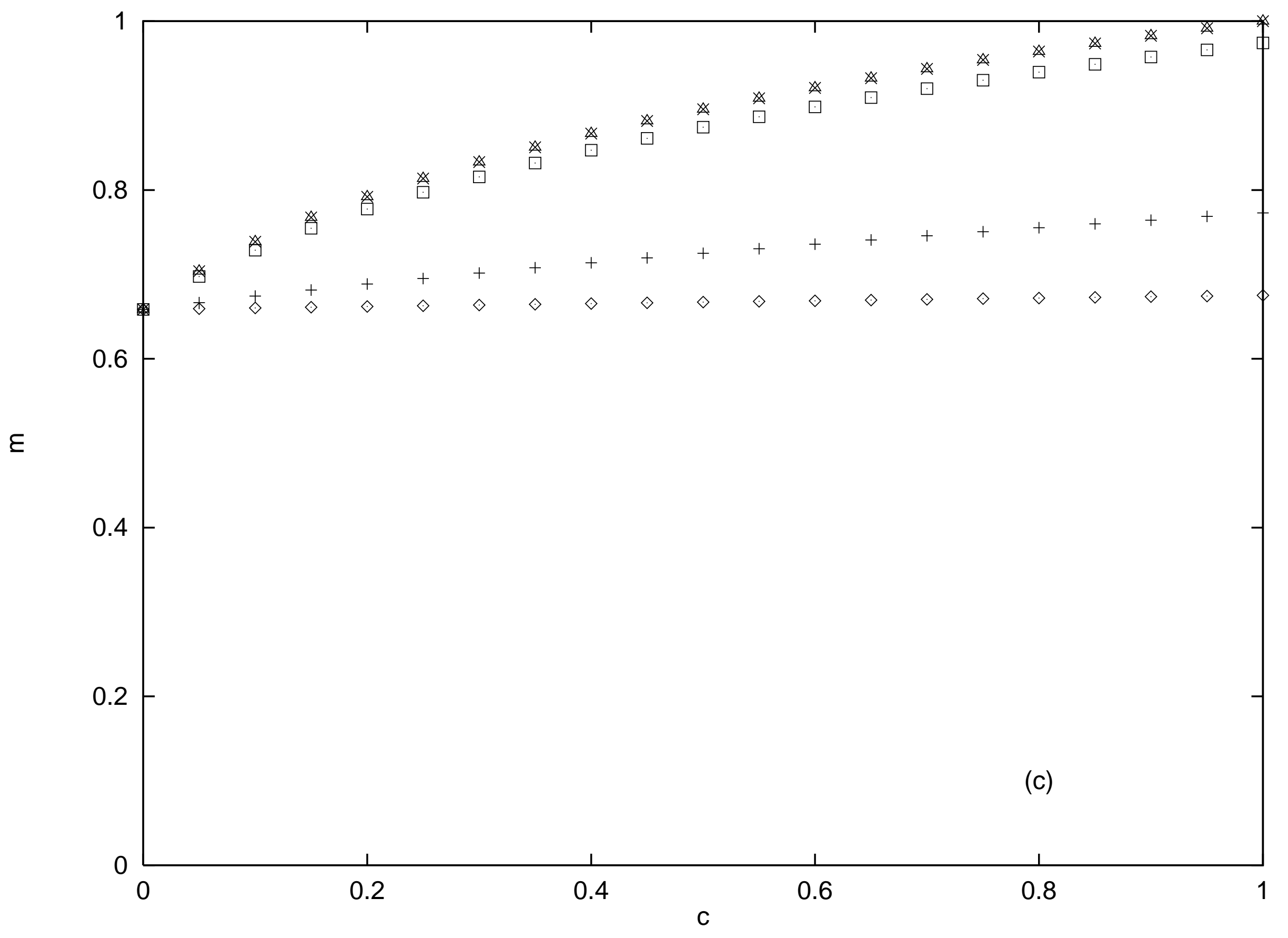

